\title{
Modification of the Acyl Chloride Quench-Labeling Method for Counting Active Sites in Catalytic Olefin Polymerization
}

\author{
Haoyang Yang ${ }^{1}$, Biao Zhang ${ }^{2, *}$, Wentao Zhong ${ }^{3}$, Zhisheng $\mathrm{Fu}^{3}$ and Zhiqiang Fan ${ }^{3, *(D)}$ \\ 1 College of Science, Zhejiang A\&F University, Hangzhou 311300, China; 20170218436@stu.zafu.edu.cn \\ 2 Zhejiang Sinopont Technology Co., Ltd., Hangzhou 311254, China \\ 3 MOE Key Laboratory of Macromolecular Synthesis and Functionalization, Department of Polymer Science \\ and Engineering, Zhejiang University, Hangzhou 310027, China; 21729017@zju.edu.cn (W.Z.); \\ fuzs@zju.edu.cn (Z.F.) \\ * $\quad$ Correspondence: zhangbiao@sinopont.com (B.Z.); fanzq@zju.edu.cn (Z.F.); Tel.: +86-572-8795-2400 (Z.F.)
}

check for updates

Citation: Yang, H.; Zhang, B.; Zhong, W.; Fu, Z.; Fan, Z. Modification of the Acyl Chloride Quench-Labeling Method for Counting Active Sites in Catalytic Olefin Polymerization. Catalysts 2021, 11, 683. https:// doi.org/10.3390/catal11060683

Academic Editor: Antonio Vittoria

Received: 13 May 2021

Accepted: 25 May 2021

Published: 28 May 2021

Publisher's Note: MDPI stays neutral with regard to jurisdictional claims in published maps and institutional affiliations.

Copyright: (c) 2021 by the authors. Licensee MDPI, Basel, Switzerland. This article is an open access article distributed under the terms and conditions of the Creative Commons Attribution (CC BY) license (https:// creativecommons.org/licenses/by/ $4.0 /)$.

\begin{abstract}
The reliable and efficient counting of active sites in catalytic olefin polymerization has been realized by using acyl chloride as the quench-labeling agent. However, the molar ratio of acyl chloride to the alkylaluminum cocatalyst must be larger than 1 in order to completely depress side reactions between the quencher and Al-polymeryl that is formed via chain transfer reaction. In this work, a tetrahydrofuran/thiophene-2-carbonyl chloride (THF/TPCC) mixture was used as the quenching agent when counting the active sites of propylene polymerization catalyzed by $\mathrm{MgCl}_{2} / \mathrm{Di} / \mathrm{TiCl}_{4}$ (Di = internal electron donor)-type Ziegler-Natta catalyst activated with triethylaluminum (TEA). When the THF/TEA molar ratio was 1 and the TPCC/TEA molar ratio was smaller than 1, the [S]/[Ti] ratio of the polymer quenched with the THF/TPCC mixture was the same as that quenched with only TPCC at TPCC/TEA > 1, indicating quench-labeling of all active sites bearing a propagation chain. The replacement of a part of the TPCC with THF did not influence the precision of active site counting by the acyl chloride quench-labeling method, but it effectively reduced the amount of acyl chloride. This modification to the acyl chloride quench-labeling method significantly reduced the amount of precious acyl chloride quencher and brought the benefit of simplifying polymer purification procedures after the quenching step.
\end{abstract}

Keywords: olefin polymerization; active sites; quench-labeling; acyl chloride; modification

\section{Introduction}

In fundamental studies on catalytic reactions, speciation and counting of the catalytic active sites play key roles, especially when the reaction mechanism is to be disclosed through systematical analysis on the reaction kinetics [1-6]. In studies on heterogeneous Ziegler-Natta (Z-N) catalysts that are widely used in the industry production of polyolefins, there is a long history of pursuing reliable and efficient methods of counting the number of polymerization active sites. For the presence of multiple active sites and their subtle changes in the reaction process, determining the number and variations of different active sites in Z-N-catalyzed olefin polymerization can provide key information for solving complicated mechanistic problems. Various quench-labeling methods have been developed in the past for counting active sites in metal-catalyzed olefin polymerization [7-23]. However, when the quenching agent simultaneously labels the metal-polymeryl bonds of the active sites and Al-polymeryl moieties formed by chain transfers with alkylaluminum cocatalysts, the corresponding quench-labeling method cannot be used to trace changes of active center concentration with reaction time. Though the average level of active site numbers can be estimated by extrapolating the $\left(\left[\mathrm{C}^{*}\right]+[\mathrm{Al}-\mathrm{P}]\right) \sim t_{\mathrm{P}}$ curve $\left(\mathrm{C}^{*}\right.$ denotes active site, $\mathrm{Al}-\mathrm{P}$ denotes Al-polymeryl moieties, $t_{\mathrm{P}}$ is the polymerization time) to $t_{\mathrm{P}}=0[11,12,15]$, the accuracy of such methods will be rather limited because of complicated kinetic behaviors in many catalytic olefin polymerizations [15]. 
In our previous works, acyl chloride ( $\mathrm{RCOCl}$ ) was used as a quenching agent to selectively label an RCO group on the end of each polymer chain that connected with the central metal of the active site in catalytic olefin polymerization [19-22]. The quenchlabeling reactions can be depicted in the following equations (see Scheme 1), wherein thiophene-2-carbonyl chloride (TPCC) was used as the quenching agent and propylene was the monomer:<smiles>CC(CP)CPC1CCCCC1C(=O)CC(C)CP</smiles>

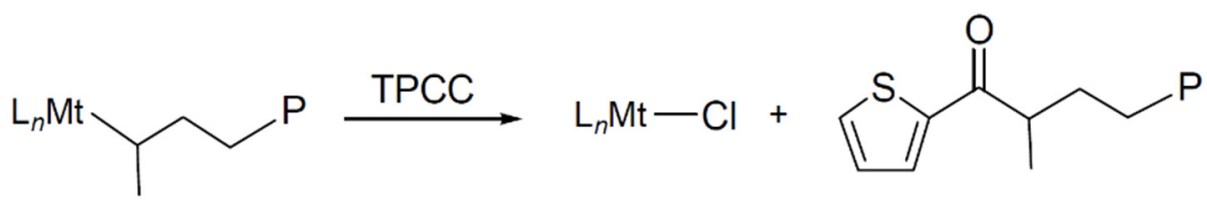<smiles></smiles>

Scheme 1. Reactions between active sites bearing propagation chains and thiophene-2-carbonyl chloride in catalytic propylene polymerization (Mt denotes central metal of the active site, and $\mathrm{L}_{n}$ denotes ligands bonded to the metal).

Since chain transfer of propagation chains with an alkylaluminum cocatalyst takes place in most catalytic olefin polymerization, the possible reaction of Al-polymeryl (the chain transfer product) with acyl chloride must be considered in acyl chloride quenchlabeling reactions (see Scheme 2). In our previous works, this side reaction was found to be completely depressed when the acyl chloride/alkylaluminum molar ratio was larger than $1[21,22,24]$. This effect could be explained by the deactivation of Al-polymeryl by excess acyl chloride. In the early literatures studying reactions between alkylaluminum or alkylaluminum chloride with acyl chloride, ketone production at high yields was realized only when the acyl chloride/alkylaluminum molar ratio was lower than $1[25,26]$. This means that alkylaluminum and acyl chloride can form a 1:1 complex that has very low reactivity with free acyl chloride molecules (see Scheme 2).

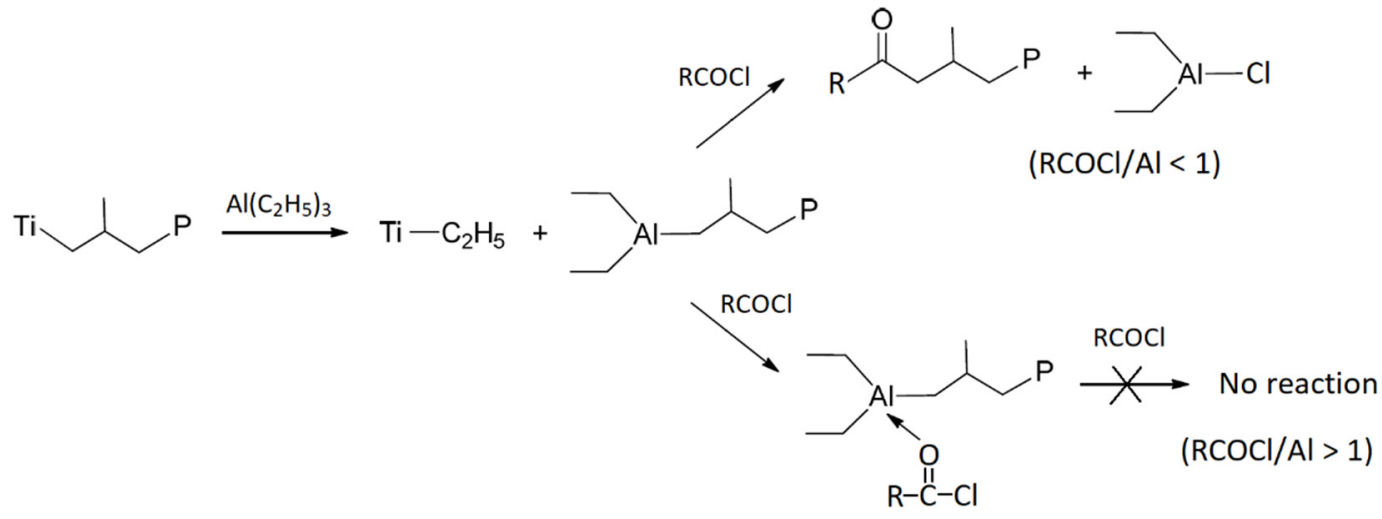

Scheme 2. Reactions between the products of chain transfers with triethylaluminum and acyl chloride.

The necessity of using excess acyl chloride quencher results in a heavy burden on polymer purification after the quenching reaction as well as waste of expensive quencher because in most catalytic olefin polymerizations, the molar ratio of alkylaluminum to 
transition metal is larger than 50. In our previous works, the molar ratio of acyl chloride quencher over transition metal in the catalyst was usually larger than 100 [19-22,24,27-29]. To reduce the amount of acyl chloride quencher in the quench-labeling system, a possible solution could be to replace a part of the quencher with an organic electron donor that did not react with the alkylaluminum cocatalyst. By using such an electron donor/acyl chloride mixture, most alkylaluminum would be deactivated by forming a complex with the donor molecule; meanwhile, the acyl chloride could still react with the chain propagation centers efficiently. In this work, tetrahydrofuran (THF) was used as an inert electron donor to replace a part of the acyl chloride in quench-labeling propylene polymerization catalyzed with a supported Z-N catalyst, and the optimal dosages of THF and acyl chloride were identified through systematical experimental studies. The chemical inertia of aliphatic ethers such as THF in contact with alkylaluminum has been proven in the literature [23]. By using a THF/acyl chloride mixture of proper composition, this work has proven the feasibility of significantly lowering the acyl chloride/alkylaluminum molar ratio without evident loss in efficiency of the quench-labeling reaction.

\section{Results and Discussion}

In the first series of experiments, parallel propylene polymerization runs in $n$-heptane slurry under 1 bar propylene pressure were conducted. A relatively large amount of $\mathrm{MgCl}_{2} / \mathrm{Di} / \mathrm{TiCl}_{4}(\mathrm{Di}=$ internal electron donor)-type Z-N catalyst $(\sim 150 \mathrm{mg})$ was used in each polymerization run, and the molar ratio of triethylaluminum (TEA) cocatalyst to titanium of the catalyst was fixed at 60 . To enhance isotacticity of the produced polypropylene, cyclohexylmethyldimethoxysilane (CHMDMS) was added at $\mathrm{Si} / \mathrm{Ti}=5$ (molar ratio) as an external electron donor [27]. At the end of 2 min polymerization, 2-thiophenecarbonyl chloride (TPCC) was added as a quenching agent to react with the Ti-polymeryl moieties of active sites and form polymer chains labeled with a 2-thiophenecarbonyl at chain end (see Scheme 1). As seen in Figure 1 and Table 1, the 4 parallel polymerization runs quenched by different amounts of TPCC (TPCC/ $\mathrm{Al}=1.2 \sim 4$ ) showed the same levels of polymer yields, meaning that they were all completely terminated by TPCC. To reduce the amount of TPCC, THF/TPCC mixtures of different compositions were used to quench the propylene polymerization conducted under the same conditions, wherein the THF/Al molar ratio was set at 1, 3 and 5, respectively. As shown in Figure 1 and Table 1, yields of these polymerization runs were the same as those quenched by only TPCC, showing that all these THF/TPCC mixtures effectively terminated the polymerization. Efficient termination of polymerization was realized by adding the THF/TPCC mixture at THF/Al $=1$ and $\mathrm{TPCC} / \mathrm{Al}=0.1$.

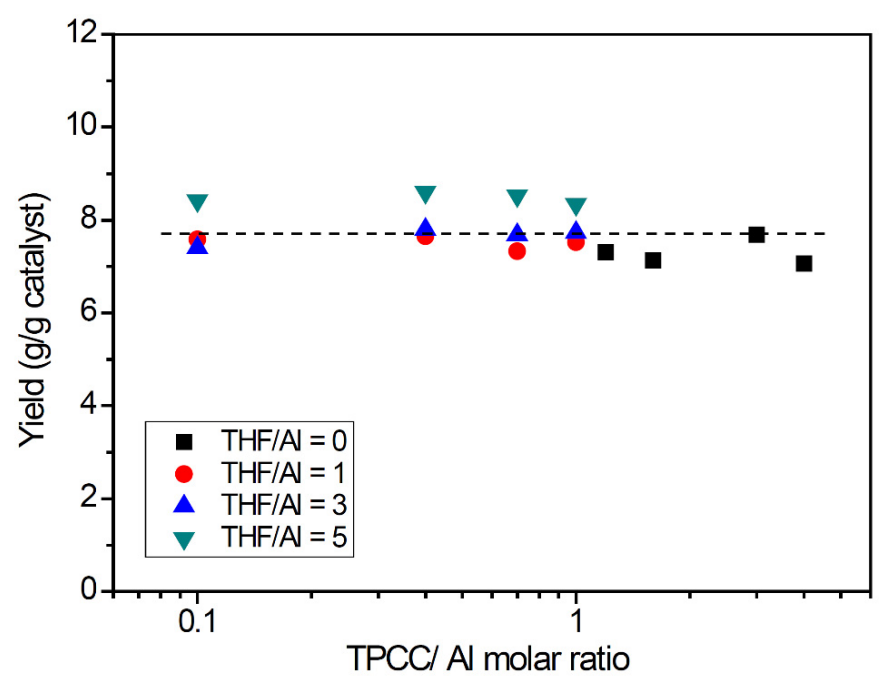

Figure 1. Effects of TPCC/Al molar ratio on yield of propylene polymerization quenched by TPCC or THF/TPCC mixture (the dotted line shows average yield of the experimental data). 
Table 1. Results of propylene polymerization quench-labeled by TPCC or THF/TPCC mixture.

\begin{tabular}{cccccc}
\hline Run & $\begin{array}{c}\text { Yield } \\
\text { (g/g Catalyst) }\end{array}$ & $\begin{array}{c}\text { Activity } \\
\mathbf{( k g / g ~ T i \cdot h )}\end{array}$ & $\begin{array}{c}\text { THF/TEA } \\
\text { (mol/mol) }\end{array}$ & $\begin{array}{c}\text { TPCC/TEA } \\
\text { (mol/mol) }\end{array}$ & $\begin{array}{c}\text { [S]/[Ti] } \\
\text { (mol\%) }\end{array}$ \\
\hline M-1 & 7.31 & 8.13 & 0 & 1.2 & 3.22 \\
M-2 & 7.13 & 7.92 & 0 & 1.6 & 2.98 \\
M-3 & 7.68 & 8.53 & 0 & 3.0 & 3.20 \\
M-4 & 7.06 & 7.85 & 0 & 4.0 & 3.18 \\
M-5 & 7.58 & 8.43 & 1 & 0.1 & 2.79 \\
M-6 & 7.65 & 8.50 & 1 & 0.4 & 2.41 \\
M-7 & 7.33 & 8.14 & 1 & 0.7 & 3.20 \\
M-8 & 7.52 & 8.35 & 1 & 1.0 & 3.19 \\
M-9 & 7.40 & 8.22 & 3 & 0.1 & 1.88 \\
M-10 & 7.80 & 8.67 & 3 & 0.4 & 1.95 \\
M-11 & 7.68 & 8.53 & 3 & 0.7 & 2.05 \\
M-12 & 7.73 & 8.59 & 3 & 1.0 & 2.32 \\
M-13 & 8.42 & 9.36 & 5 & 0.1 & 1.81 \\
M-14 & 8.60 & 9.55 & 5 & 0.4 & 1.47 \\
M-15 & 8.53 & 9.48 & 5 & 0.7 & 1.83 \\
M-16 & 8.34 & 9.26 & 5 & 1.0 & 2.10 \\
\hline
\end{tabular}

(a) THF was mixed with TPCC first, and then the mixture was added to the polymerization system.

The efficiency of the quench-labeling reaction was evaluated by measuring the sulfur content of the polypropylene (PP) product after thorough purification. As seen in Table 1 and Figure 2, the sulfur content of the polymer samples quenched by only TPCC was concentrated at around $[\mathrm{S}] /[\mathrm{Ti}]=3.2 \mathrm{~mol} \%$ when the TPCC $/ \mathrm{Al}$ ratio was changed from 1.2 to 4 , just as in the phenomena reported in our previous work [22]. It meant that quantitative quenching of all Ti-polymeryl species was realized when the TPCC/Al ratio was larger than 1, and possible reactions between TPCC and Al-polymeryl species are depressed, for each alkylaluminum molecule is deactivated through complexation with a TPCC molecule (see Scheme 2). Since the number of the un-complexed or "free" acyl chloride exceeded the number of Ti-polymeryl species when the TPCC/Al ratio was larger than 1.1, completeness of the quenching reaction could be guaranteed. In the case of the quenching reaction at $\mathrm{TPCC} / \mathrm{Al}=1.2$ (Run M-1), the molar ratio of "free" TPCC to Ti was only 12.

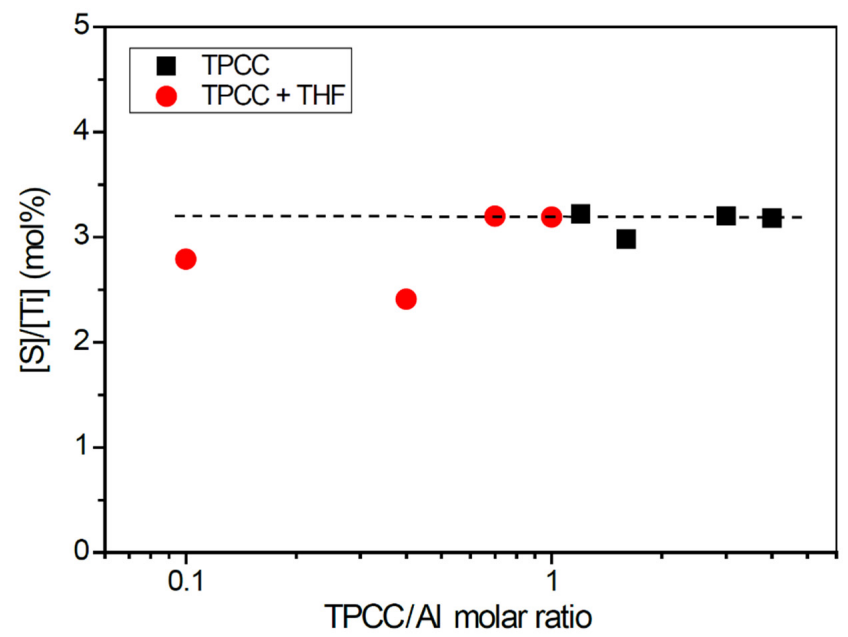

Figure 2. Effects of TPCC/Al molar ratio on sulfur content of polymers quench-labeled by TPCC or $\mathrm{THF} / \mathrm{TPCC}$ mixture $(\mathrm{THF} / \mathrm{Al}=1)$. (The dotted line shows average levels of the $[\mathrm{S}] /[\mathrm{Ti}]$ ratio from the quenching experiments with only TPCC.)

When the THF/TPCC mixture was used as the quenching agent, the $[\mathrm{S}] /[\mathrm{Ti}]$ fractions of the polymer fell at the same level of those quenched by only TPCC when THF/Al ratio was fixed at 1 (see Figure 2), but further increase of THF/Al to 3 or 5 resulted in lower 
[S]/[Ti] fractions (see Table 1). It meant that the role of excess TPCC in forming a 1:1 complex with $\mathrm{AlEt}_{3}$ could be replaced by THF at THF/Al = 1, so the TPCC/ $\mathrm{Al}$ molar ratio could be reduced to about 0.7 without sacrificing the efficiency of the quench-labeling reaction. However, when the THF/Al ratio was increased to 3 or even 5, the efficiency of the TPCC quenching was significantly depressed. Even when the TPCC/Al ratio was as high as 1, the THF/TPCC mixture with THF/ $\mathrm{Al}>1$ still gave an evidently lower [S]/[Ti] value. This negative effect of THF on the quenching reaction could be explained by the competition of un-complexed THF with TPCC in coordination on the active sites, as THF (a mono-ether) has a strong tendency for coordinating on the Lewis-acidic active sites. It seemed that THF had a stronger tendency for coordinating on alkylaluminum than TPCC, so most THF could have coordinated with TEA to form a 1:1 complex, leaving very little un-complexed THF that could compete with TPCC in coordinating with the active sites. At $\mathrm{THF} / \mathrm{Al}>1$, a large number of un-complexed THF molecules would seriously compete with TPCC and reduce the efficiency of the quenching reaction (see Scheme 3).

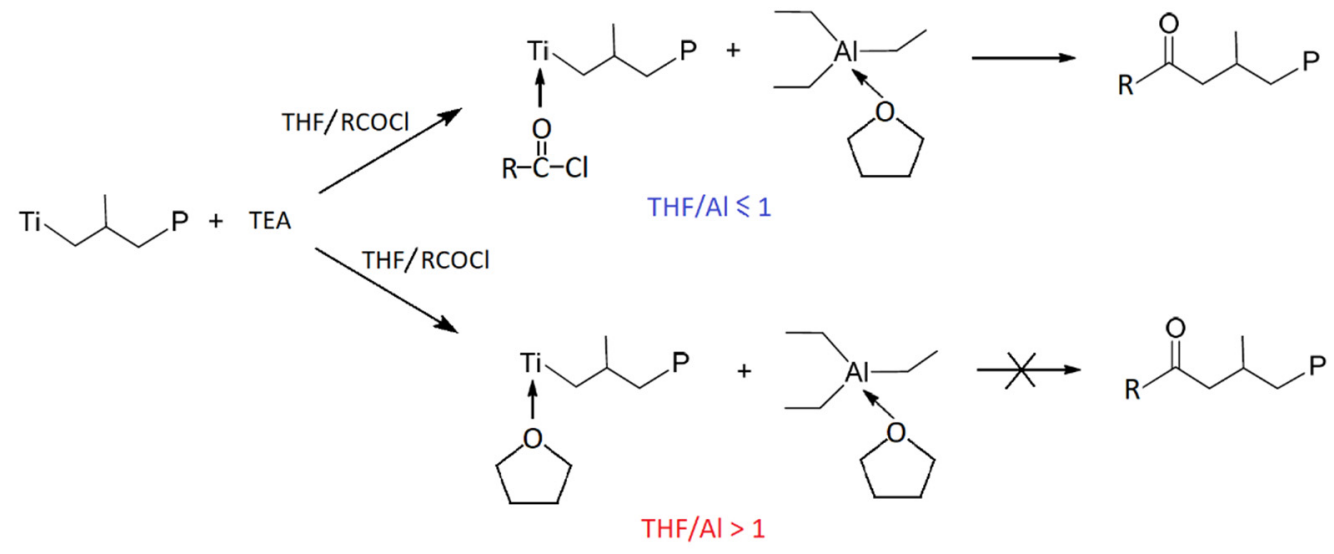

Scheme 3. Coordination of THF with alkylaluminum and active sites in a quenching reaction using a THF/acyl chloride mixture as the quenching agent.

Another type of quenching experiment was also studied in which the THF solution was injected into the propylene polymerization system first, and the TPCC solution was added 2 min later. It was expected that the THF would form a complex with TEA and the alkylaluminum that had Al-polymeryl moiety before the addition of TPCC. As seen in Table 2 and Figure 3, adding THF before TPCC caused markedly lower [S]/[Ti] values as compared with the results of adding only TPCC. Even when the THF/Al ratio was 1, lower $[\mathrm{S}] /[\mathrm{Ti}]$ was still observed. It seemed that a high proportion of active sites were coordinated by THF when it was added before TPCC. Compared with the un-complexed $\mathrm{C}^{*}$, the $\mathrm{C}^{*}$ /THF complex had much lower reactivity in contact with TPCC, resulting in an incomplete quenching reaction.

Table 2. Results of propylene polymerization quenched by separately added THF and TPCC.

\begin{tabular}{cccccc}
\hline Run & $\begin{array}{c}\text { Yield } \\
\text { (g/g Catalyst) }\end{array}$ & $\begin{array}{c}\text { Activity } \\
(\mathbf{k g} / \mathbf{g T i} \cdot \mathbf{h})\end{array}$ & $\begin{array}{c}\text { THF/TEA } \\
(\mathbf{m o l} / \mathbf{m o l})\end{array}$ & $\begin{array}{c}\text { TPCC/TEA } \\
(\mathbf{m o l} / \mathbf{m o l})\end{array}$ & $\begin{array}{c}\text { [S]/[Ti] } \\
(\mathbf{m o l} \%)\end{array}$ \\
\hline S-1 & 8.37 & 9.30 & 1 & 0.1 & 0.60 \\
S-2 & 8.11 & 9.02 & 1 & 0.4 & 0.63 \\
S-3 & 8.19 & 9.10 & 1 & 0.7 & 1.08 \\
S-4 & 7.14 & 7.94 & 1 & 1.0 & 1.08 \\
S-5 & 7.25 & 8.06 & 3 & 0.1 & 0.46 \\
S-6 & 7.79 & 8.65 & 3 & 0.4 & 0.61 \\
S-7 & 7.89 & 8.76 & 3 & 0.7 & 0.95 \\
S-8 & 7.76 & 8.62 & 3 & 1.0 & 1.47 \\
\hline
\end{tabular}

(a) THF was added 2 min before the addition of TPCC. 


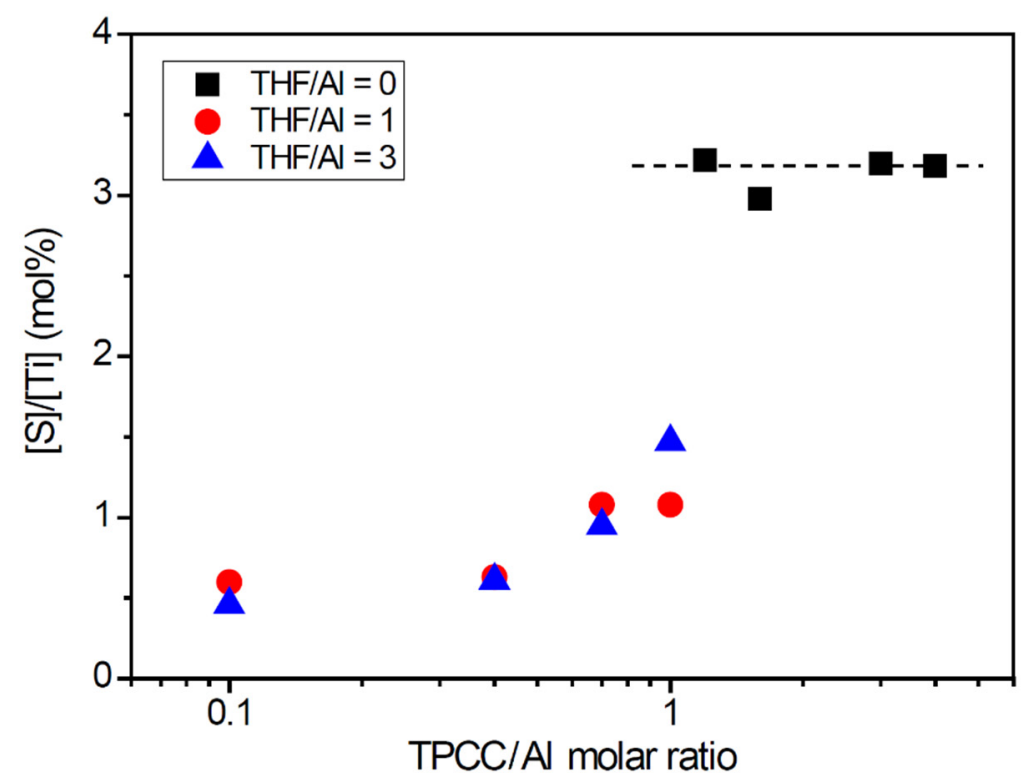

Figure 3. Effects of TPCC/Al molar ratio on sulfur content of polymer quench-labeled by separately added THF and TPCC. (The dotted line shows the average level of [S]/[Ti] ratio from the quenching experiments with only TPCC.)

To find more evidence for understanding the observed phenomena, a part of the polymer samples in Tables 1 and 2 were extracted by boiling $n$-heptane, and the sulfur contents of the insoluble fractions were determined. This kind of extraction method has been widely used in academic and industrial research to separate isotactic PP chains from those with lower stereoregularity [27]. The fraction that is insoluble in boiling $n$-heptane is composed of isotactic PP that has high crystallinity and a high melting temperature [27]. As seen in Table 3, the samples had similar values of C7-ins content (content of isotactic $\mathrm{PP}$, or isotacticity index) because they were synthesized under the same conditions. The ([S]/[Ti] $)_{\text {ins }}$ values in Table 3 are the number of thiophenecarbonly-labeled isotactic PP chains, which were produced by the parts of active sites with high stereospecificity. It was natural that the ([S]/[Ti]) ins values were smaller than $[S] /[T i]$, the total number of labeled PP chains. When only TPCC was used as the quenching agent, the $[S]_{\text {ins }} /[S]$ ratio (fraction of isospecific active sites in the catalyst) was around $50 \%$, close to the values found in our previous work $[27,30]$. When THF/TPCC mixtures were used and THF/Al ratio was 1 (runs M-5 and M-7), similar [S] $]_{\text {ins }} /[S]$ fractions were observed, showing that these quenching conditions did not influence quenching of either the isospecific or the non-stereospecific active sites by TPCC. However, when THF/TPCC mixtures were used at THF/Al $>1$ (run M-11) or THF was added before TPCC (run S-6), not only was the total number of labeled PP chains ([S]/[Ti]) lowered, but the ([S] $\left.]_{\text {ins }} /[\mathrm{S}]\right)$ ratio was also evidently lowered. It meant that under these quenching conditions, the reaction of TPCC and isospecific active sites was depressed by THF more than that of TPCC and non-stereospecific active sites. In other words, the complexes of isospecific active sites and THF were more stable than those of non-stereospecific active sites and THF. This difference between the two groups of active sites could be explained by the larger degree of spatial congestion in the isospecific active sites. As reported in the literature, the isospecific active sites of supported Z-N catalyst contain more atoms or adsorbates in their second coordination sphere, making their stereochemical environment more crowded than non-stereospecific sites [27,31]. The THF coordinated on such crowded sites will have a lower probability of leaving the active site via coordinating to Lewis-acidic $\mathrm{AlR}_{3}$ in the solution. Therefore, the different stabilities between isospecific site-THF complex and non-stereospecific site-THF complex could be taken as further evidence for elucidating the nature of the negative effects of THF on the TPCC quench-labeling reaction. It is possible that more selective blocking of the stereospecific active sites could be realized by using inert electron donors with suitable 
bulkiness. By combining such electron donors with the acyl chloride quencher, a new method of characterizing the active site distribution of $\mathrm{Z}-\mathrm{N}$ catalysts could be developed.

Table 3. Fractions of boiling heptane insoluble parts of PP samples and their sulfur contents.

\begin{tabular}{|c|c|c|c|c|c|c|c|}
\hline Sample & $\begin{array}{c}\text { Activity } \\
(\mathrm{kg} / \mathrm{gTi} \cdot \mathrm{h})\end{array}$ & $\begin{array}{l}\text { THF/TEA } \\
(\mathrm{mol} / \mathrm{mol})\end{array}$ & $\begin{array}{l}\text { TPCC/TEA } \\
\text { (mol/mol) }\end{array}$ & $\begin{array}{c}\text { C7-ins } \\
\left.(w)^{(a)}\right)\end{array}$ & $\begin{array}{c}([\mathrm{S}] /[\mathrm{Ti}])^{(\mathrm{b})} \\
(\mathrm{mol} \%)\end{array}$ & $\begin{array}{c}([\mathrm{S}] /[\mathrm{Ti}])_{\mathrm{ins}}(\mathrm{c}) \\
(\mathrm{mol} \%)\end{array}$ & $\begin{array}{c}{[\mathrm{S}]_{\text {ins }} /[\mathrm{S}]} \\
(\mathrm{mol} / \mathrm{mol})\end{array}$ \\
\hline M-3 & 8.53 & 0 & 0 & 87.1 & 3.20 & 1.73 & 0.54 \\
\hline M-5 & 8.43 & 1 & 0.1 & 88.7 & 2.79 & 1.72 & 0.62 \\
\hline M-7 & 8.14 & 1 & 0.7 & 86.5 & 3.20 & 2.06 & 0.64 \\
\hline M-11 & 8.53 & 3 & 0.7 & 89.2 & 2.05 & 0.88 & 0.43 \\
\hline S-6 & 8.65 & $3^{(\mathrm{d})}$ & 0.4 & 90.2 & 0.61 & 0.19 & 0.31 \\
\hline
\end{tabular}

(a) Weight percentages of boiling $n$-heptane insoluble fractions; (b) sulfur contents of the whole PP samples; ${ }^{(\mathrm{c})}$ sulfur contents of the PP fractions insoluble in boiling $n$-heptane; ${ }^{(\mathrm{d})}$ THF was added 2 min before adding TPCC.

The different THF effects between two modes of quenching agent additions (addition of THF/TPCC mixture vs. separate addition of THF and TPCC) implied that the complexes of THF and active sites were thermodynamically more stable than those of TPCC and active sites. However, quenching of the active sites by TPCC seemed to be kinetically more favored, so complete quenching of all active sites could be realized by adding THF/TPCC mixture to the catalysis system when THF/ Al ratio was not larger than 1.

\section{Materials and Methods}

Materials: $\mathrm{A} \mathrm{MgCl}_{2} / \mathrm{Di} / \mathrm{TiCl}_{4}(\mathrm{Di}=$ diol ester, Ti content $=2.7 \mathrm{wt} \%$ )-type Z-N catalyst was kindly donated by SINOPEC and used for propylene polymerization. Polymerizationgrade propylene (purchased from Hangzhou Minxing Chemical Technology Co., Ltd., Hangzhou, China) was purified by passing it through a column packed with deoxygen reagent and 4 A molecular sieves. Triethylaluminum (TEA, purchased from Albemarle) and 2-thiophenecarbonyl chloride (TPCC, purchased from J\&K Scientific) were diluted with $n$ heptane to $2 \mathrm{~mol} / \mathrm{L}$ before use. Cyclohexylmethyldimethoxysilane (CHMDMS) (purchased from Linyi Lujing Chemical Co., Linyi, China) was purified by vacuum distillation and diluted in $n$-heptane to $0.25 \mathrm{~mol} / \mathrm{L}$ before use. $n$-Heptane (Shanghai Titan Scientific Co., Ltd., Shanghai, China), used as the solvent, was dried over $4 \AA$ A molecular sieves, refluxed over sodium metal for $6 \mathrm{~h}$ and distilled before use. Ethanol ( $99.5 \%)$ and $\mathrm{HCl}$ ( $36 \%$ solution) were purchased from Hangzhou Chemical Reagents Co. (Hangzhou, China) and used as received.

Polymerization and quench-labeling: Propylene polymerizations were conducted in a $100 \mathrm{~mL}$ flask equipped with a magnetic stirring bar. The flask was dried by heating under vacuum and refilling with nitrogen 3 times, and designated amounts of $n$-heptane $(\sim 60 \mathrm{~mL})$, $\operatorname{AlEt}_{3}(\mathrm{Al} / \mathrm{Ti}=60)$ and CHMDMS $(\mathrm{Si} / \mathrm{Ti}=5)$ were successively introduced into the flask under a propylene atmosphere of $1 \mathrm{~atm}$ at $60^{\circ} \mathrm{C}$. The catalyst powder (about $150 \mathrm{mg}$ ) was then added to start polymerization at $60^{\circ} \mathrm{C}$, during which propylene gas at $1 \mathrm{~atm}$ was continuously supplied to the flask. After $2 \mathrm{~min}$, TPCC solution in $n$-heptane or THF/TPCC mixture was injected into the flask to quench the polymerization, and the liquid phase was continuously stirred at $60^{\circ} \mathrm{C}$ for $5 \mathrm{~min}$. In another type of quenching experiment, a designated amount of THF solution was first injected into the flask and stirred for $2 \mathrm{~min}$, and then a designated amount of TPCC solution was injected to quench-label the active sites carrying propagation chains for $5 \mathrm{~min}$. After the quench-labeling reaction, the liquid phase in the flask was poured into the excess ethanol/ $\mathrm{HCl}$ mixture $(95 / 5)$ to decompose the catalyst, cocatalyst and quencher, and the produced polypropylene (PP) was precipitated as fine powder. The filtered PP product was repeatedly washed with ethanol and dried at $60{ }^{\circ} \mathrm{C}$ under vacuum for $6 \mathrm{~h}$.

Purification of the polymer after quenching: The PP samples were further purified for accurate sulfur content measurement. The same procedures of sample purification as reported in our previous work [27] were adopted. 
Solvent extraction of the polypropylene product: Weighed PP sample was extracted with boiling $n$-heptane for $12 \mathrm{~h}$ in Soxhlet extractor, and the insoluble part was dried and weighed.

Analytical methods: Sulfur content of each PP sample and the boiling $n$-heptane insoluble part of PP was measured in a YHTS-2000 ultraviolet fluorescence sulfur analyzer (Jiangyan Yinhe Instrument Co., Jiangyan, China). About 2 4 mg polymer sample was weighed to $\pm 0.01 \mathrm{mg}$ and used in the analysis, and the average value of three parallel measurements was taken as the sulfur content. The [S]/[Ti] molar ratio was calculated according to the following equation:

$$
[\mathrm{S}] /[\mathrm{Ti}]=\left(S_{\mathrm{p}} \times m_{\mathrm{p}} / 32 \times 10^{6}\right) /\left(m_{\mathrm{c}} \times 0.027 / 47,900\right)
$$

where $S_{\mathrm{p}}$ is sulfur content $(\mathrm{ppm}), m_{\mathrm{p}}$ is weight of polymerization product $(\mathrm{g}), m_{\mathrm{c}}$ is weight of catalyst $(\mathrm{mg})$.

\section{Conclusions}

By using THF/TPCC mixture as the quenching agent and controlling the THF/Al molar ratio to no higher than 1 , active sites in propylene polymerization with a Z-N catalyst could be quantitatively and efficiently quench-labeled by an acyl chloride quencher, even though the TPCC/Al molar ratio was reduced to 0.7. In contrast, the TPCC/Al molar ratio could not be lower than 1 when only TPCC was used as the quencher. This modification to the acyl chloride quench-labeling method thus significantly reduced the amount of acyl chloride quencher and brought benefits such as simplifying polymer purification procedures after the quenching step and saving precious quenching agent.

Author Contributions: Methodology, investigation, formal analysis, data curation, H.Y., W.Z. and B.Z.; resources, Z.F. (Zhisheng Fu) and Z.F. (Zhiqiang Fan); writing-original draft preparation, B.Z. and H.Y.; writing - review and editing, conceptualization, supervision, project administration, Z.F. (Zhiqiang Fan); funding acquisition, Z.F. (Zhiqiang Fan), Z.F. (Zhisheng Fu) and B.Z. All authors have read and agreed to the published version of the manuscript.

Funding: This research was funded by the National Natural Science Foundation of China (grant number 51773178) and the Zhejiang Postdoctoral Science Foundation (grant number zj2019049).

Conflicts of Interest: The authors declare no conflict of interest. The funders had no role in the design of the study; in the collection, analyses, or interpretation of data; in the writing of the manuscript; or in the decision to publish the results.

\section{References}

1. Tuttle, R.R.; Folkman, S.J.; Rubin, H.N.; Finke, R.G.; Reynolds, M.M. Copper metal-organic framework surface catalysis: Catalyst poisoning, IR spectroscopic, and kinetic evidence addressing the nature and number of the catalytically active sites en route to improved applications. ACS Appl. Mater. Inter. 2020, 12, 39043-39055. [CrossRef] [PubMed]

2. Razdan, N.K.; Bhan, A. Carbidic Mo is the sole kinetically-relevant active site for catalytic methane dehydroaromatization on Mo/H-ZSM-5. J. Catal. 2020, 389, 667-676. [CrossRef]

3. Kondrat, S.A.; van Bokhoven, J.A. A perspective on counting catalytic active sites and rates of reaction using X-Ray spectroscopy. Top. Catal. 2019, 62, 1218-1227. [CrossRef]

4. Desert, X.; Carpentier, J.-F.; Kirillov, E. Quantification of active sites in single-site group 4 metal olefin polymerization catalysis. Coord. Chem. Rev. 2019, 386, 50-68. [CrossRef]

5. Damaskinos, C.M.; Vasiliades, M.A.; Stathopoulos, V.N.; Efstathiou, A.M. The effect of $\mathrm{CeO}_{2}$ preparation method on the carbon pathways in the dry reforming of methane on $\mathrm{Ni} / \mathrm{CeO}_{2}$ studied by transient techniques. Catalysts 2019, 9, 621. [CrossRef]

6. Egbert, J.D.; Lopez-Ruiz, J.A.; Prodinger, S.; Holladay, J.D.; Mans, D.M.; Wade, C.E.; Weber, R.S. Counting surface redox sites in carbon-supported electrocatalysts by cathodic stripping of $\mathrm{O}$ deposited from $\mathrm{N}_{2} \mathrm{O}$. J. Catal. 2018, 365, 405-410. [CrossRef]

7. Cipullo, R.; Mellino, S.; Busico, V. Identification and count of the active sites in olefin polymerization catalysis by oxygen quench. Macromol. Chem. Phys. 2014, 215, 1728-1734. [CrossRef]

8. Natta, G.; Pasquon, I. The kinetics of the stereospecific polymerization of alpha-olefins. Adv. Catal. 1959, 11, 1-66.

9. Chumaevskii, N.B.; Zakharov, V.A.; Bukatov, G.D.; Kuznetzova, G.I.; Yermakov, Y.I. Study of mechanism of propagation and transfer-reactions in polymerization of olefins by Ziegler-Natta catalysts, 1 Determination of number of propagation centers and rate constant. Makromol. Chem. Macromol. Chem. Phys. 1976, 177, 747-761. [CrossRef] 
10. Chien, J.C.W. Kinetics of ethylene polymerization catalyzed by bis-(cyclopentadienyl)-titanium dichloride dimethylaluminum chloride. J. Am. Chem. Soc. 1959, 81, 86-92. [CrossRef]

11. Chien, J.C.W.; Tsai, W.M. Zirconocenium cation catalysis of propene polymerization. Makromol. Chem. Macromol. Symp. 1993, 66, 141-156. [CrossRef]

12. Feldman, C.F.; Perry, E. Active centers in the polymerization of ethylene using titanium tetrachloride-alkylaluminum catalysts. J. Polym. Sci. 1960, 46, 217-231. [CrossRef]

13. Mejzlik, J.; Lesna, M.; Kratochvila, J. Determination of the number of active centers in Ziegler-Natta polymerizations of olefins. Adv. Polym. Sci. 1987, 81, 83-120.

14. Vozka, P.; Mejzlík, J. Determination of the number of active-centers in Ziegler-Natta polymerization of propene using sulfurcontaining terminating agents, 1 Application of carbon-disulfide. Makromol. Chem. 1989, 190, 1489-1499. [CrossRef]

15. Marques, M.M.; Tait, P.J.T.; Mejzlik, J.; Dias, A.R. Polymerization of ethylene using high-activity Ziegler-type catalysts: Active center determination. J. Polym. Sci. Part A Polym. Chem. 1998, 36, 573-585. [CrossRef]

16. Liu, Z.X.; Somsook, E.; Landis, C.R. A ${ }^{2} \mathrm{H}$-labeling scheme for active-site counts in metallocene-catalyzed alkene polymerization. J. Am. Chem. Soc. 2001, 123, 2915-2916. [CrossRef]

17. Nelsen, D.L.; Anding, B.J.; Sawicki, J.L.; Christianson, M.D.; Arriola, D.J.; Landis, C.R. Chromophore quench-labeling: An approach to quantifying catalyst speciation as demonstrated for (EBI) $\mathrm{ZrMe}_{2} / \mathrm{B}\left(\mathrm{C}_{6} \mathrm{~F}_{5}\right)_{3}$-catalyzed polymerization of 1-hexene. ACS Catal. 2016, 6, 7398-7408. [CrossRef]

18. Cueny, E.S.; Johnson, H.C.; Anding, B.J.; Landis, C.R. Mechanistic studies of hafnium-pyridyl amido-catalyzed 1-octene polymerization and chain transfer using quench-labeling methods. J. Am. Chem. Soc. 2017, 139, 11903-11912. [CrossRef]

19. Fan, Z.Q.; Feng, L.X.; Yang, S.L. Study on the mechanism of 1-octene Ziegler-Natta polymerization based on the number of active centers. Chin. J. Polym. Sci. 1991, 9, 113-119.

20. Fan, Z.Q.; Feng, L.X.; Yang, S.L. Distribution of active centers on $\mathrm{TiCl}_{4} / \mathrm{MgCl}_{2}$ catalyst for olefin polymerization. J. Polym. Sci. Part A Polym. Chem. 1996, 34, 3329-3335. [CrossRef]

21. Zhang, L.T.; Fu, Z.S.; Fan, Z.Q. A new method of active center determination for olefin polymerization with supported ZieglerNatta catalysts. Macromol. Res. 2010, 18, 695-700. [CrossRef]

22. Shen, X.R.; Hu, J.; Fu, Z.S.; Lou, J.Q.; Fan, Z.Q. Counting the number of active centers in $\mathrm{MgCl}_{2}$-supported Ziegler-Natta catalysts by quenching with 2-thiophenecarbonyl chloride and study on the initial kinetics of propylene polymerization. Catal. Commun. 2013, 30, 66-69. [CrossRef]

23. Yu, Y.; Cipullo, R.; Boisson, C. Alkynyl ether labeling: A selective and efficient approach to count active sites of olefin polymerization catalysts. ACS Catal. 2019, 9, 3098-3103. [CrossRef]

24. Yang, P.J.; Zhong, W.T.; Jiang, B.Y.; Zhang, B.; Fu, Z.S.; Fan, Z.Q. Determination and tracing of active and dormant propagation chains in 1-hexene polymerization with supported Ziegler-Natta catalyst. Appl. Catal. A Gen. 2020, 595, 117469. [CrossRef]

25. Adkins', H.; Scanle, C. Ketones from acyl chlorides and alkyl aluminum chlorides: A selective keto-ester synthesis. J. Am. Chem. Soc. 1951, 73, 2854-2856. [CrossRef]

26. Spielmann, W.; Schaeffer, G. Process for the Manufacture of Ketones. U.S. Patent US4,266,066A, 5 May 1985.

27. Shen, X.R.; Fu, Z.S.; Hu, J.; Wang, Q.; Fan, Z.Q. Mechanism of propylene polymerization with $\mathrm{MgCl}_{2}$-supported Ziegler-Natta catalysts based on counting of active centers: The role of external electron donor. J. Phys. Chem. C 2013, 117, 15174-15182 [CrossRef]

28. Guo, Y.T.; Zhang, Z.; Guo, W.Q.; Khan, A.; Fu, Z.S.; Xu, J.T.; Fan, Z.Q. Kinetics and mechanism of metallocene catalyzed olefin polymerization: Comparison of ethylene, propylene homopolymerizations and their copolymerization. J. Polym. Sci. Part A Polym. Chem. 2017, 55, 867-875. [CrossRef]

29. Jiang, B.Y.; Liu, X.Y.; Weng, Y.H.; Fu, Z.S.; He, A.H.; Fan, Z.Q. Mechanistic study on comonomer effect in ethylene/1-hexene copolymerization with $\mathrm{TiCl}_{4} / \mathrm{MgCl}_{2}$ model Ziegler-Natta catalysts. J. Catal. 2019, 369, 324-334. [CrossRef]

30. Zhang, B.; Qian, Q.Y.; Yang, P.J.; Jiang, B.Y.; Fu, Z.S.; Fan, Z.Q. Responses of supported Ziegler-Natta catalyst to comonomer feed ratio in ethylene-propylene copolymerization: Differentiation of active centers with different catalytic features. Ind. Eng. Chem. Res. 2021, 60, 4575-4588. [CrossRef]

31. Vittoria, A.; Meppelder, A.; Friederichs, N.; Busico, V.; Cipullo, R. Demystifying Ziegler-Natta catalysts: The origin of stereoselectivity. ACS Catal. 2017, 7, 4509-4518. [CrossRef] 